\title{
Anatomical and metabolic small- animal whole-body imaging using ring-shaped confocal photoacoustic computed tomography
}

Jun Xia, Muhammad Chatni, Konstantin Maslov, Lihong V. Wang

Jun Xia, Muhammad Chatni, Konstantin Maslov, Lihong V. Wang, "Anatomical and metabolic small-animal whole-body imaging using ringshaped confocal photoacoustic computed tomography," Proc. SPIE 8581, Photons Plus Ultrasound: Imaging and Sensing 2013, 85810K (4 March 2013); doi: 10.1117/12.2004937

SPIE. Event: SPIE BiOS, 2013, San Francisco, California, United States 


\title{
Anatomical and metabolic small-animal whole-body imaging using ring-shaped confocal photoacoustic computed tomography
}

\author{
Jun Xia, Muhammad Chatni, Konstantin Maslov, and Lihong V. Wang* \\ Department of Biomedical Engineering, One Brookings Drive, Washington University in St. Louis, \\ St. Louis, MO, USA 63130 \\ *Correspondence should be addressed tolhwang@biomed.wustl.edu
}

\begin{abstract}
Due to the wide use of animals for human disease studies, small animal whole-body imaging plays an increasingly important role in biomedical research. Currently, none of the existing imaging modalities can provide both anatomical and glucose metabolic information, leading to higher costs of building dual-modality systems. Even with image coregistration, the spatial resolution of the metabolic imaging modality is not improved. We present a ring-shaped confocal photoacoustic computed tomography (RC-PACT) system that can provide both assessments in a single modality. Utilizing the novel design of confocal full-ring light delivery and ultrasound transducer array detection, RC-PACT provides full-view cross-sectional imaging with high spatial resolution. Scanning along the orthogonal direction provides three-dimensional imaging. While the mouse anatomy was imaged with endogenous hemoglobin contrast, the glucose metabolism was imaged with a near-infrared dye-labeled 2-deoxyglucose. Through mouse tumor models, we demonstrate that RC-PACT may be a paradigm shifting imaging method for preclinical research.
\end{abstract}

Keywords: Photoacoustic computed tomography, small-animal whole-body imaging, metabolic imaging

\section{INTRODUCTION}

The importance of imaging tumor metabolism establishes itself from the premise that anatomical changes alone are not an accurate metric for diagnosis, prognosis, and therapy. In particular, tumor metabolic imaging provides a spatial and temporal map of therapeutic response and guides treatment planning and selection. Therefore, simultaneous metabolic and anatomical imaging is very desirable, and necessary not only in clinical settings, but also in preclinical cancer models to improve our understanding of cancer, metastasis, and drug efficacy in vivo [1,2].

Currently, X-ray computed tomography (CT) [3] and magnetic resonance imaging (MRI) [4] provide high-resolution anatomical images, but they cannot provide sufficient metabolic contrast without combining with metabolic imaging modalities. Despite the high cost of dual-modality imaging systems and the need for image registration, metabolic imaging systems have their own limitations. For instance, positron emission tomography (PET) is very expensive, and the radioisotopes are difficult to manufacture and have short half-lives. In addition, the accumulated radiation dosage of the combined PET/CT may be carcinogenic and will confound results in oncology $[5,6]$.

Here, we present a ring-shaped confocal photoacoustic computed tomography (RC-PACT) scanner that can image both anatomy and tumor glucose metabolism at high spatial resolution in a single imaging modality [7, 8]. In RC-PACT, anatomical images were produced by endogenous hemoglobin contrast, and tumor glucose metabolism was imaged and quantified using spectral separation of the absorption of IRDye800-2DG, a near-infrared fluorophore-labeled glucose analog, from that of hemoglobin [9]. With the unique design of confocal free-space full-ring light illumination and fullring ultrasound transducer array detection, our RC-PACT system generates in vivo cross-sectional images with simultaneous anatomical and metabolic assessments at high spatial resolution [10].

\section{SYSTEM DESIGN}

Figure 1 shows the schematic of the RC-PACT system [11]. A tunable Ti-Sapphire laser with $12 \mathrm{~ns}$ pulse duration and $10 \mathrm{~Hz}$ pulse repetition rate was used as the irradiation source. The laser beam passed through a conical lens to form a ring-shaped light, which was then focused using an optical condenser to project a thin light band around the object. The light incident area was aligned to be slightly above the acoustic focal plane to minimize the detection of strong surface

Photons Plus Ultrasound: Imaging and Sensing 2013, edited by Alexander A. Oraevsky, Lihong V. Wang, Proc. of SPIE Vol. 8581, 85810K - (C) 2013 SPIE · CCC code: 1605-7422/13/\$18 - doi: 10.1117/12.2004937 
signals. The photoacoustic signals were detected by a 512-element full-ring transducer array with $5 \mathrm{MHz}$ central frequency ( $80 \%$ bandwidth) and $50 \mathrm{~mm}$ ring diameter. Each element in the array was mechanically shaped into an arc to produce an axial focal depth of $19 \mathrm{~mm}$ within the imaging plane. The combined foci of all elements generated a central imaging region of $20 \mathrm{~mm}$ diameter and $1 \mathrm{~mm}$ thickness [11, 12]. Since the light incidence was oblique, the light formed a weak focus inside the animal body. This focal region overlapped with the acoustic focal plane to improve the efficiency of detecting photoacoustic signals generated in deep tissues [7].

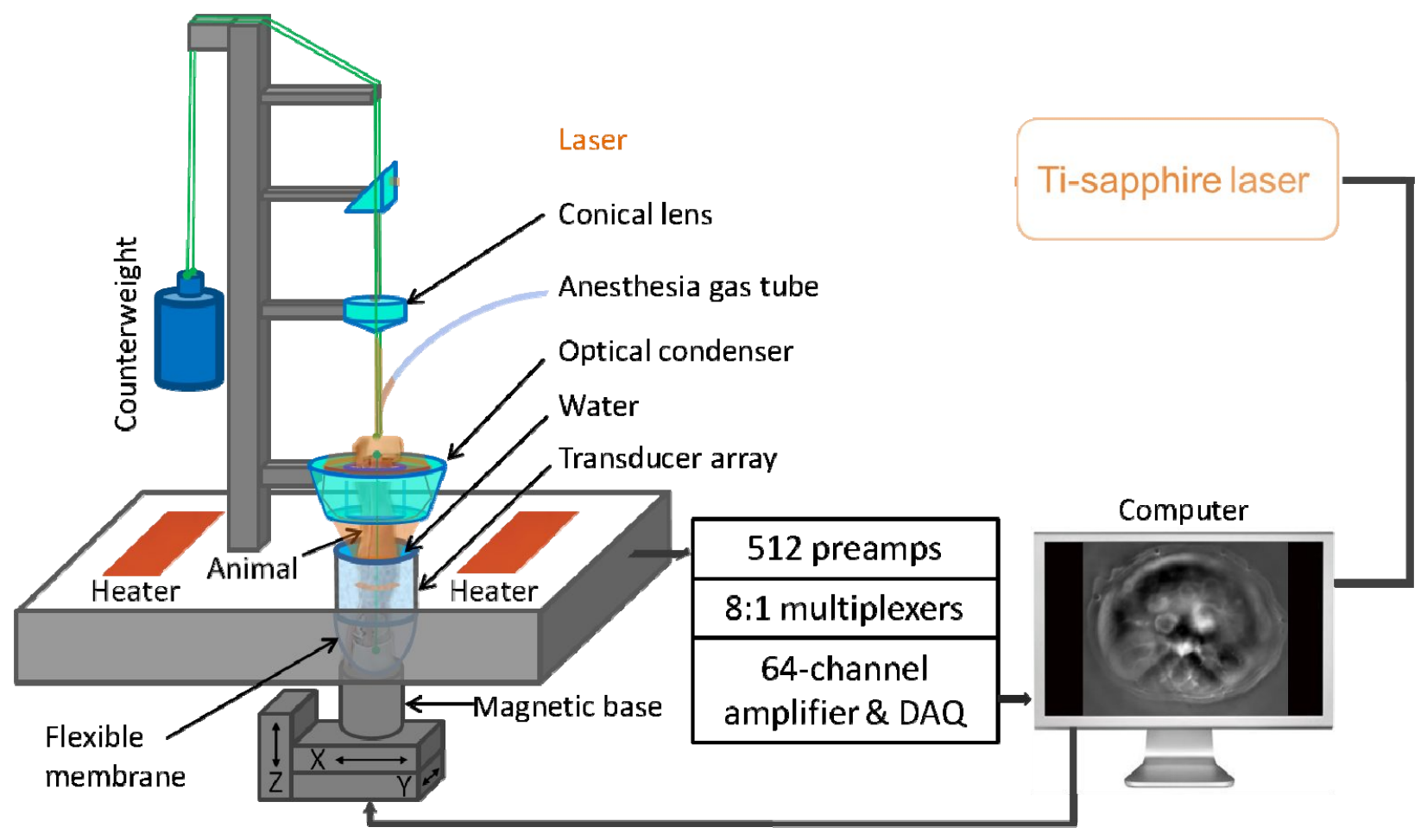

Figure 1. Experimental setup of the full-ring confocal whole-body photoacoustic computed tomography system.

\section{SYSTEM CALIBRATION}

In spectral experiments, the beam profile of the laser varied at different wavelengths, which led to changes in the fluence distribution, adding complexities on spectral separation. This problem can be solved either by aligning the optics after each wavelength tuning, which requires a tremendous amount of time in three-dimensional scanning, or more efficiently, by post-experimental compensation. In this research, we used a phantom calibration method to compensate for both the laser power and the light band uniformity of different wavelengths. The phantom was made from a gelatin-water suspension mixed with $0.1 \%$ black ink. The suspension was put in a cylindrical container, whose inner diameter was similar to the mouse cross-sectional diameter $(20 \mathrm{~mm})$, and was then cooled in the fridge until gelled. The gelled phantom was then mounted on the RC-PACT scanner and imaged using the same scheme as the animal experiment. Cross-sectional photoacoustic images of the phantom were reconstructed at each wavelength, and the ring-shaped light variation was obtained based on the surface signal of the phantom image. The matrix of light variation was then used as a weighting function to calibrate the spectral in vivo images. The calibration results are demonstrated in Figure 2 . The compensated images have similar signal intensity at different wavelengths and a more uniform signal distribution around the illumination ring. 

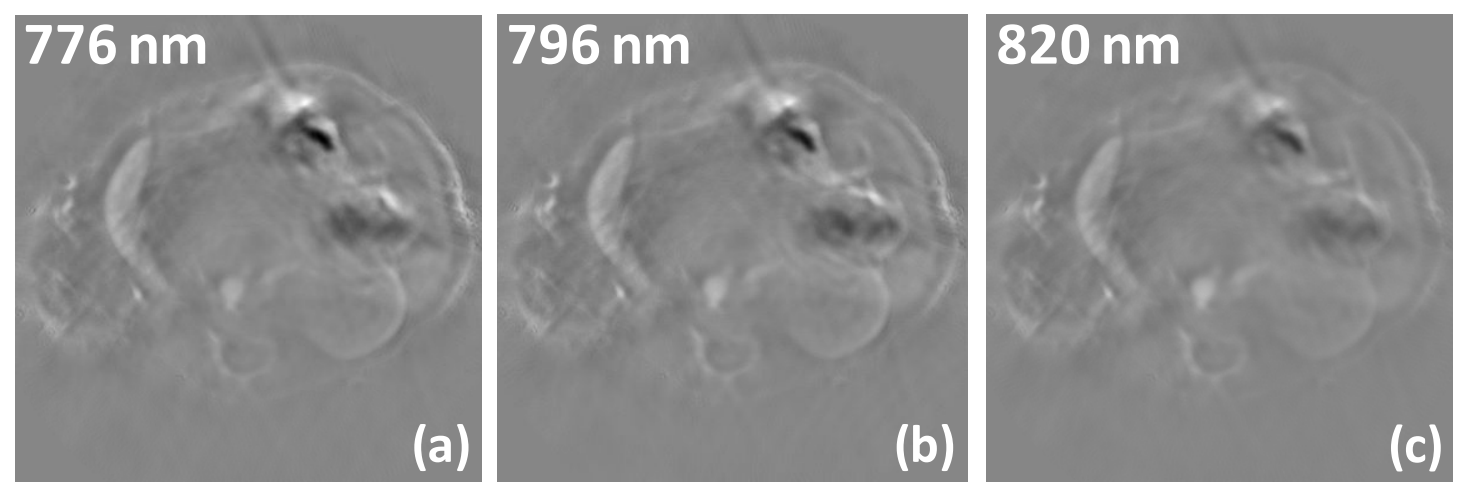

$\operatorname{Max}$
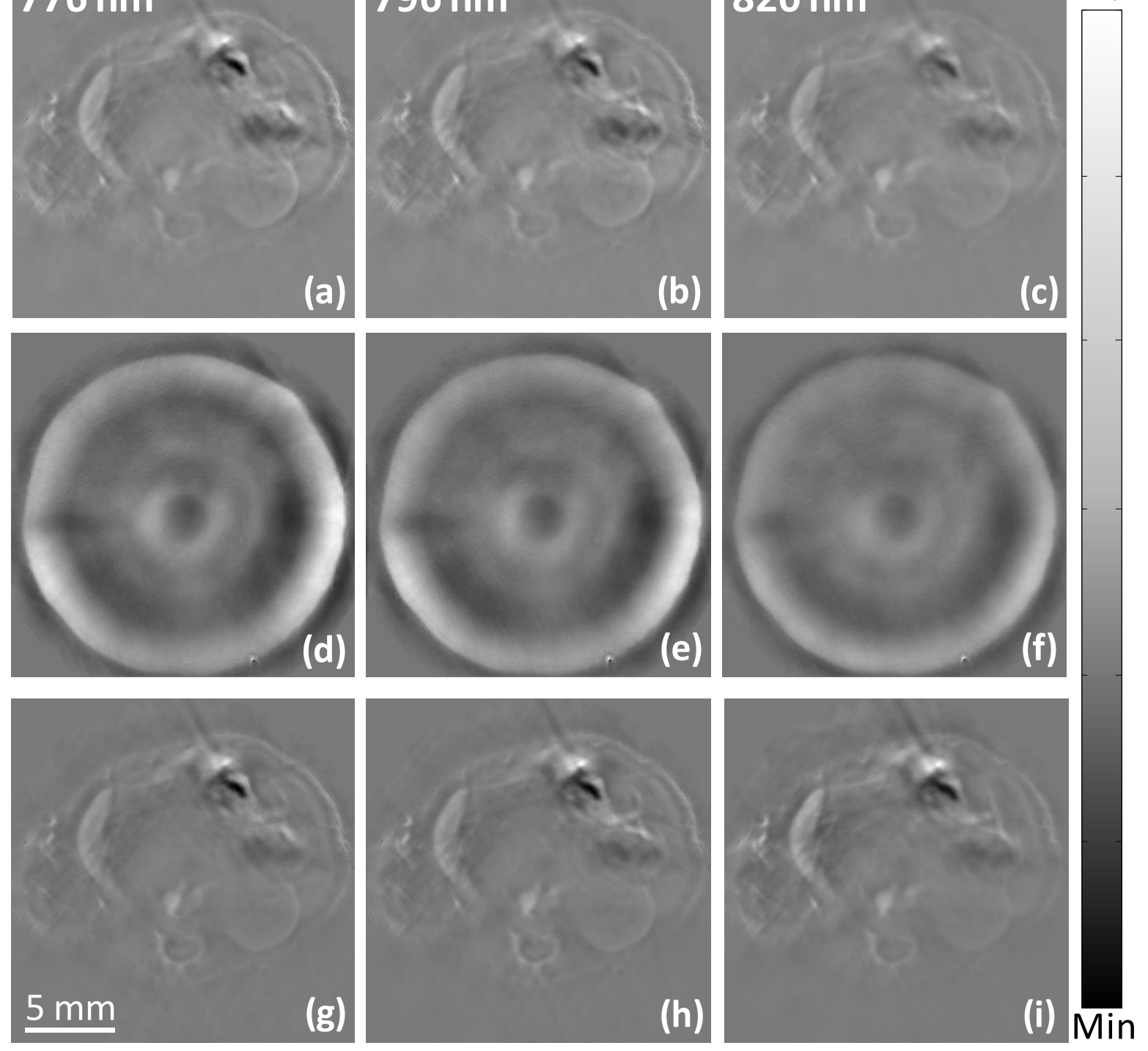

Figure 2. Image compensation for the wavelength-dependent laser power and ring-shaped-light distribution. Top row (a-c), uncompensated images. Middle row (d-f), phantom images. Bottom row (g-i), compensated images. Left column: images acquired at $776 \mathrm{~nm}$. Middle column: images acquired at $796 \mathrm{~nm}$. Right column: images acquired at $820 \mathrm{~nm}$.

\section{IN VIVO ANATOMICAL IMAGING}

To demonstrate the in vivo anatomical imaging capability of our RC-PACT system, we imaged healthy athymic (nude) mice. Figure 3 shows serial cross-sectional images of 5-6-week-old healthy athymic mice in axial views. The brain cortex, heart, liver, spleen, and kidneys are clearly visible. Additionally, detailed vascular structures within these organs are visible, demonstrating that the system can be used for whole-body angiographic imaging without injecting exogenous contrasts. The spinal cord, stomach, and gastrointestinal tracts are imaged due to the surrounding microvasculature. Major blood vessels, such as the vena cava, are also clearly visible. Using exogenous optical contrast (e.g., near-infrared dyes), the system can also image organs with little blood. The bladder image was acquired 30 minutes after tail vein administration of IRDye800-2DG. The urinary bladder showed strong contrast as it was filled with the dye excreted by the kidneys. 


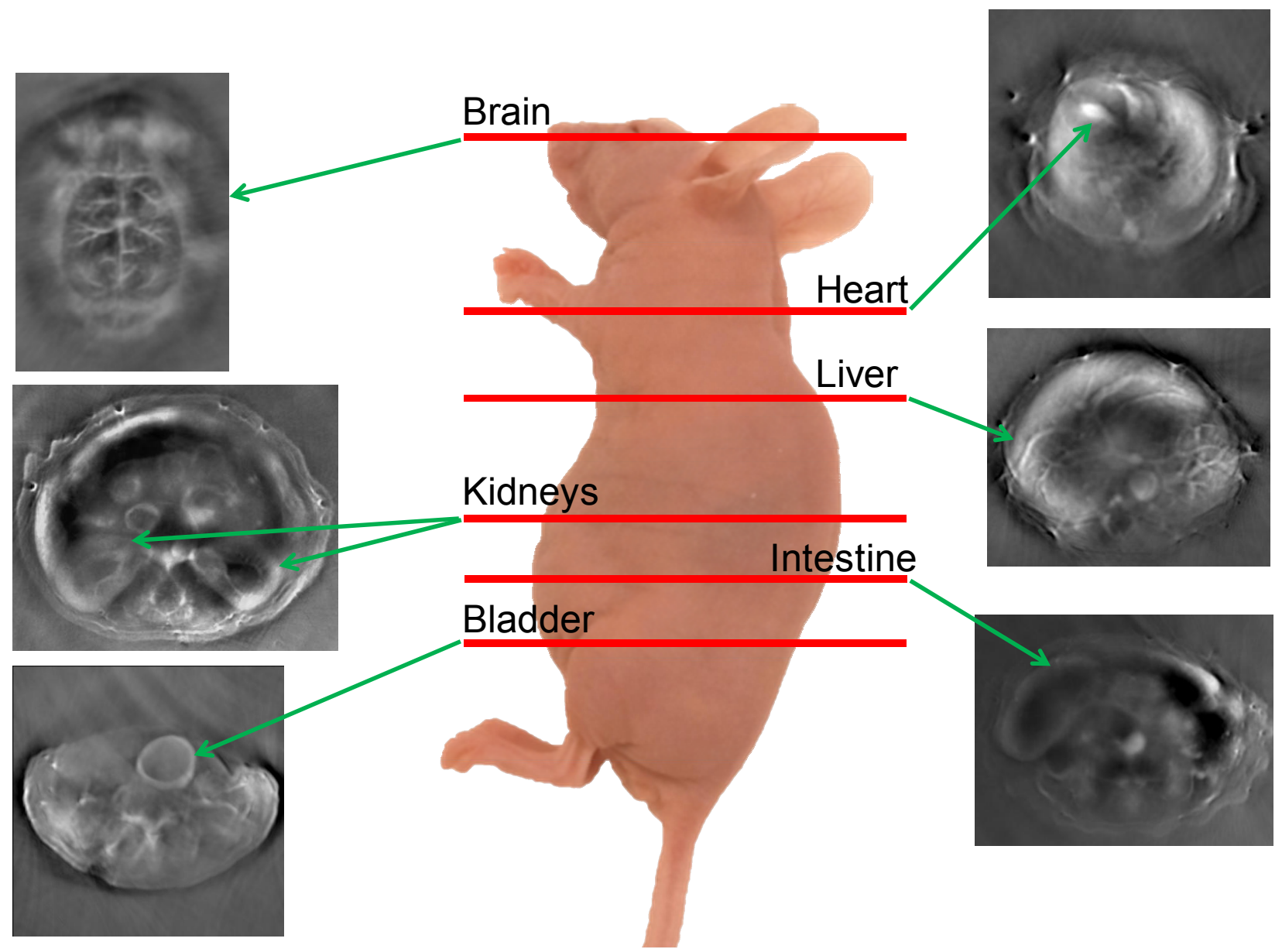

Figure 3. RC-PACT images of athymic mice acquired noninvasively at various anatomical locations:

\section{IN VIVO TUMOR GLUCOSE METABOLISM IMAGING}

To showcase the capability of our RC-PACT system to image the glucose metabolism of deeper tumors, we imaged two mice with orthotopically implanted 786-O tumor cells in their left kidneys.

Following the control experiment, $200 \mu \mathrm{L}$ of $(0.4 \mathrm{mM})$ IRDye $800-2 \mathrm{DG}$ was administered, and the mice were imaged after 24 hours. This 24-hour time window is sufficient for excess unbound IRDye800-2DG to be excreted by the kidneys and bladder. This also increases the likelihood that the imaged IRDye800-2DG reflects tumor glucose metabolism rather than the physical entrapment of excess dye molecules inside the tumors. Figures 4(a) show representative anatomical cross-sections of the two 786-O tumor mice. The $\mathrm{HbT}$ concentrations were normalized by the maximum $\mathrm{HbT}$ concentration (observed in the vena cava), scaled to $2.3 \mathrm{mM}$ [Figures 4(b)], and then the absolute IRDye800-2DG concentrations in the anatomical cross-section were estimated. Images of tumor glucose metabolism [figures 4(c)] were overlaid on the gray-scale PACT images acquired at $776 \mathrm{~nm}$. In Figures 4(c), the mean IRDye800-2DG concentration in healthy kidneys were used as the threshold to account for non-specific IRDye800-2DG uptake due to kidneys being the excretory route. The signal-to-noise ratio for imaging IRDye800-2DG in the most metabolically active site in the tumor was $39.3 \mathrm{~dB}$ for mouse 1 and $38.3 \mathrm{~dB}$ for mouse 2. The tumor-to-normal tissue contrast for IRDye800-2DG uptake was approximately 3.3 at the most metabolically active site in the tumor. The same image reconstruction and spectral separation methods were also applied on the control RC-PACT images, and no significant IRDye800-2DG uptake was observed. 

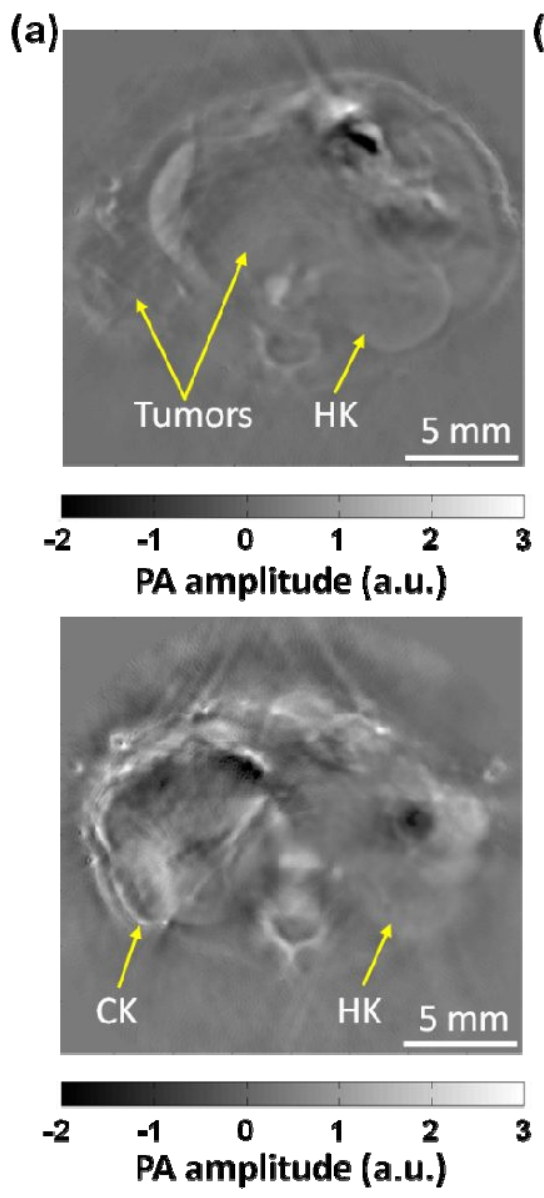

(b)
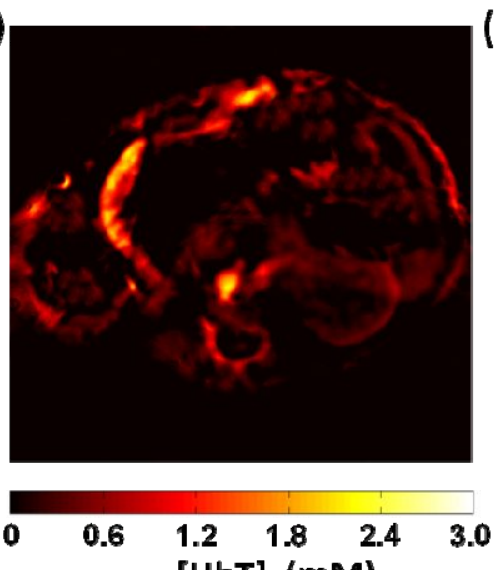

[HbT] (mM)

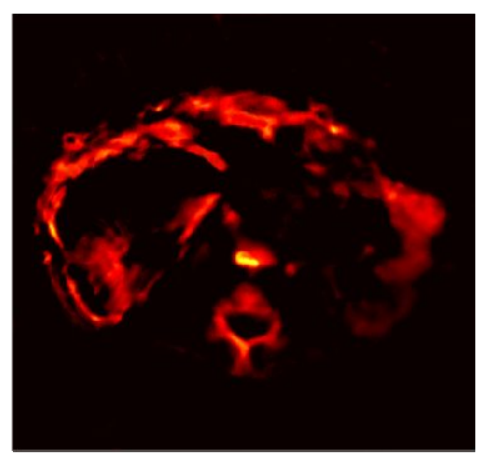

$\begin{array}{lllll}0.6 & 1.2 & 1.8 & 2.4 & 3.0\end{array}$ [HbT] (mM)
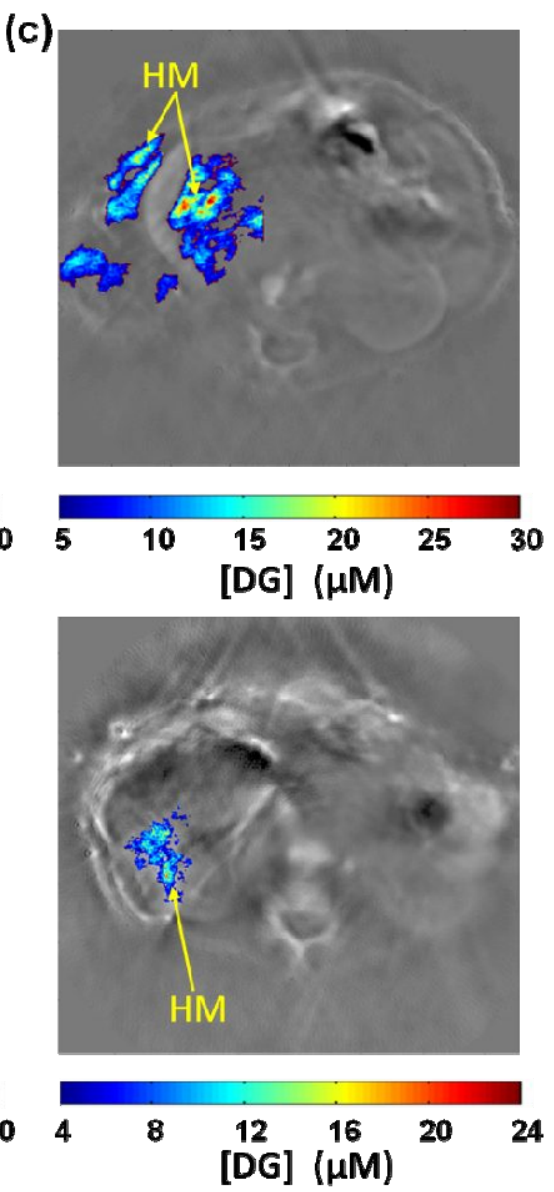

Figure 4. In vivo RC-PACT images of orthotopically implanted 786-O kidney tumors. Top row: mouse 1. Bottom row: mouse 2. (a) Anatomical images acquired at $776 \mathrm{~nm}$. (b) HbT images overlaid on the anatomical images. (c) IRDye800-2DG images overlaid on the anatomical images. CK, cancerous kidney; HK, healthy kidney; HM, hypermetabolic.

\section{DISCUSSION AND CONCLUSION}

Anatomical and metabolic imaging is of great importance due to its wide application in tumor biology, drug discovery, and early-stage cancer screening in both clinical and pre-clinical settings. In this report, we have demonstrated that RCPACT provided tumor glucose metabolism and anatomy images with ultrasound-defined spatial resolution in a single imaging modality. While PET-CT and PET-MRI have been well established in the field, RC-PACT is a promising and less expensive alternative with the advantages of non-ionizing laser radiation, non-radioactive optical contrast, and endogenous hemoglobin contrast. Additionally, image co-registration of anatomical and metabolic assessments is automatic. Besides imaging tumor glucose metabolism, RC-PACT can also benefit from near-infrared fluorescent dyes [8] and proteins [13] for deep molecular imaging along with detailed, high resolution anatomical structures. The possibility of multiplexing functional parameters in combination with anatomical imaging uniquely positions RC-PACT to have a broad impact in preclinical medical research and life sciences. Due to the fixed ring diameter of the current system, we only demonstrated $1 \mathrm{~cm}$ penetration depth; however, photoacoustic imaging at depth of $8.4 \mathrm{~cm}$ has been 
reported [14]. Taking advantage of the full-ring light illumination, which doubles the imaging depth $(\sim 16.8 \mathrm{~cm})$, RCPACT can potentially be scaled to image larger animals or even humans.

\section{ACKNOWLEDGEMENTS}

The authors appreciate Ms. Seema Dahlheimer's close reading of the manuscript. This work was sponsored in part by National Institutes of Health grants R01 EB000712, R01 EB008085, R01 CA134539, U54 CA136398, R01 EB010049, R01 CA157277, R01 CA159959, and 5P60 DK02057933. L.W. has financial interests in Microphotoacoustics, Inc. and Endra, Inc., which, however, did not support this work.

\section{REFERENCES}

[1] M. Baker, "Whole-animal imaging: The whole picture," Nature, 463(7283), 977-980 (2010).

[2] R. A. de Kemp, F. H. Epstein, C. Catana et al., "Small-Animal Molecular Imaging Methods," Journal of Nuclear Medicine, 51(Supplement 1), 18S-32S (2010).

[3] D. W. Holdsworth, and M. M. Thornton, "Micro-CT in small animal and specimen imaging," Trends in Biotechnology, 20(8), S34-S39 (2002).

[4] H. Benveniste, and S. Blackband, "MR microscopy and high resolution small animal MRI: applications in neuroscience research," Progress in Neurobiology, 67(5), 393-420 (2002).

[5] G. Brix, U. Lechel, G. Glatting et al., "Radiation Exposure of Patients Undergoing Whole-Body Dual-Modality 18F-FDG PET/CT Examinations," Journal of Nuclear Medicine, 46(4), 608-613 (2005).

[6] R. Fazel, H. M. Krumholz, Y. Wang et al., "Exposure to Low-Dose Ionizing Radiation from Medical Imaging Procedures," New England Journal of Medicine, 361(9), 849-857 (2009).

[7] J. Xia, M. Chatni, K. Maslov et al., "Whole-body ring-shaped confocal photoacoustic computed tomography of small animals in vivo," Journal of Biomedical Optics, 17(5), 050506 (2012).

[8] J. Yao, J. Xia, K. I. Maslov et al., "Noninvasive photoacoustic computed tomography of mouse brain metabolism in vivo," NeuroImage, 64, 257-266 (2013).

[9] J. L. Kovar, W. Volcheck, E. Sevick-Muraca et al., "Characterization and performance of a near-infrared 2deoxyglucose optical imaging agent for mouse cancer models," Analytical Biochemistry, 384(2), 254-262 (2009).

[10] M. R. Chatni, J. Xia, R. Sohn et al., "Tumor glucose metabolism imaged in vivo in small animals with wholebody photoacoustic computed tomography," Journal of Biomedical Optics, 17(7), 076012 (2012).

[11] J. Xia, Z. Guo, K. Maslov et al., "Three-dimensional photoacoustic tomography based on the focal-line concept," Journal of Biomedical Optics, 16(9), 090505 (2011).

[12] J. Gamelin, A. Maurudis, A. Aguirre et al., "A real-time photoacoustic tomography system for small animals," Opt. Express, 17(13), 10489-10498 (2009).

[13] G. S. Filonov, A. Krumholz, J. Xia et al., "Deep-Tissue Photoacoustic Tomography of a Genetically Encoded Near-Infrared Fluorescent Probe," Angewandte Chemie International Edition, 51(6), 1448-1451 (2012).

[14] H. Ke, T. N. Erpelding, L. Jankovic et al., "Performance characterization of an integrated ultrasound, photoacoustic, and thermoacoustic imaging system," Journal of Biomedical Optics, 17(5), 056010-1 (2012). 